Claremont Colleges

Scholarship@ Claremont

Pomona Faculty Publications and Research

Pomona Faculty Scholarship

$1-1-1992$

\title{
Construction of silicon nanocolumns with the scanning tunneling microscope
}

David M. Tanenbaum

Pomona College

R.M. Ostrom

Alan C. Gallagher

\section{Recommended Citation}

The following article appeared in "Construction of silicon nanocolumns with the scanning tunneling microscope," R. M. Ostrom, D. M. Tanenbaum, and Alan Gallagher, Appl. Phys. Lett. 61, 925 (1992), DOI:10.1063/1.107730 and may be found at http://link.aip.org/link/?APPLAB/61/925/1 


\title{
Construction of silicon nanocolumns with the scanning tunneling microscope
}

\author{
R. M. Ostrom, D. M. Tanenbaum, and Alan Gallagher ${ }^{\text {a) }}$ \\ Joint Institute for Laboratory Astrophysics, National Institute of Standards and Technology \\ and University of Colorado, Boulder, Colorado 80309-0440
}

(Received 28 February 1992; accepted for publication 8 June 1992)

\begin{abstract}
Voltage pulses to a scanning tunneling microscope (STM) are used to construct silicon columns of 30-100 $\AA$ diameter and up to $200 \AA$ height on a silicon surface and on the end of a tungsten probe. These nanocolumns have excellent conductivity and longevity, and they provide an exceptional new ability to measure the shapes of nanostructures with a STM. This construction methodology and these slender yet robust columns provide a basis for nanoscale physics, lithography, and technology.
\end{abstract}

As described in recent reviews, ${ }^{1,2}$ the potential of the scanning tunneling microscope (STM) to induce surface modifications has been investigated for some time. Initial attempts generally produced relatively large area and erratic surface changes using direct probe-surface contact or large energy deposition in a small area, but finer features and even sliding individual physisorbed $\mathrm{Xe}$ atoms along a $\mathrm{Ni}$ surface to controlled positions ${ }^{3}$ have now been achieved. Nanometer scale surface-layer chemical changes, ${ }^{4}$ surface etching, ${ }^{5}$ chemical vapor deposition, ${ }^{6}$ deposition of probe material, ${ }^{7}$ and field induced atom transfer have all been demonstrated. ${ }^{8,9}$

The atom-sliding manipulations ${ }^{3}$ were done with the probe within angstroms of the $\mathrm{Xe}$ atom, and the result was independent of the sign of the probe bias. Consequently, the forces which allow this exacting position control were ascribed to a combination of electrostatic and van der Waals interactions. Recently, Eigler et al. have also demonstrated that $\mathrm{Xe}$ atoms can be repeatedly transferred back and forth between probe and sample, apparently due to an electromigration force. ${ }^{10}$ Whitman et al. have shown that Cs atoms physisorbed onto GaAs will slide from quite large distances $(\sim 1000 \AA)$ toward a negatively biased probe. ${ }^{11}$ As explained in Refs. 2 and 11 the Cs manipulations result from the interaction of the probe-substrate electric field with the surface induced and field induced dipole moments of the physisorbed Cs atoms. The surfaceinteraction dipole moment corresponds to displacing the electron $1 \AA$ toward the surface, so the Cs atoms are preferentially attracted toward a negative probe. Lyo and Avouris have shown that $\mathrm{Si}$ atoms can be transferred to a negatively biased probe in near contact $(1-3 \AA$ ) with a Si surface and back to a different surface site with reverse bias. ${ }^{9}$ In this case chemically assisted field evaporation of $\mathrm{Si}$ atoms is the suggested cause. Note that field evaporation involves the same field-dipole force as in surface sliding; both act on surface $\mathrm{Si}$ with a positive dipole moment.

The atom-sliding techniques described above have manipulated only atoms of the adsorbed surface layer. Similarly, atom transfer between sample and probe, whether

\footnotetext{
a'Staff Member, Quantum Physics Division, National Institute of Standards and Technology.
}

near contact ${ }^{8,9}$ or electromigration, ${ }^{10}$ has only dealt with physisorbed atoms or the first few atomic layers of the surface. Gold mounds of $\sim 20 \AA$ height and $150 \AA$ width have been reproducibly placed on a gold surface, ${ }^{7}$ but nanostructures with the high aspect ratios (height/width) characteristic of microelectronics have not been achieved. Here we report the construction of thin Si nanocolumns up to $200 \AA$ tall, and with height/diameter $>1$. These $\mathrm{Si}$ nanocolumns have been grown on the probe as well as at repeated locations on the Si sample. We believe that the mechanism for these manipulations involves primarily the field-dipole interaction described by Whitman et al. for Cs on GaAs. However, in the present case this interaction dislocates surface atoms and slides them across the surface, then stacks up $\mathrm{Si}$ atoms into a nanocolumn below the probe. A Si nanocolumn also grows slowly on the W probe by Si atom transfer from the sample column. This transfer is attributed to a combination of electrostatic and chemical forces, similar to the transfers in Ref. 9.

We use etched $W$ probes that are thermally cleaned in ultrahigh vacuum (UHV) and $\sim 0.01 \Omega n$-type $\mathrm{Si}(100$ ) or $\mathrm{Si}(111)$. The samples are heated in UHV to desorb the oxide layer that results from a standard Shiraki etch cleaning. ${ }^{12} \mathrm{~A}$ sharp $2 \times 1$ or $7 \times 7$ low energy electron diffraction (LEED) pattern confirms the initial cleanliness and reconstruction of the $\mathrm{Si}$ surface. The pattern acquires a diffuse background after a few days in the UHV chamber. The results shown here are obtained without noticeable change on samples that have been in the UHV chamber for $1 \mathrm{~h}$ to 1 week since oxide removal, confirming that they are intrinsic to $\mathrm{Si}$ and not related to any surface contaminants. Construction is done on surface regions that are initially atomically flat, aside from occasional atomic steps.

Nanocolumn formation has been done by a variety of techniques, such as ramping the sample bias to $7 \mathrm{~V}$ in $\sim 1$ $\mathrm{s}$ or applying $6-8 \mathrm{~V}$ to the sample for $1-10 \mathrm{~s}$ while maintaining current feedback. In most cases we have repeatedly pulsed or ramped the voltage at one position, each time referencing the probe from the top of the existing structure and subsequently scanning the region at $3 \mathrm{~V}$ and $0.1 \mathrm{nA}$ to assess changes. Nanocolumns are normally grown on the sample by pulsing the sample positive. We have found it necessary to fuse the probe to the sample once with a brief, large voltage pulse before we could achieve a sufficiently 


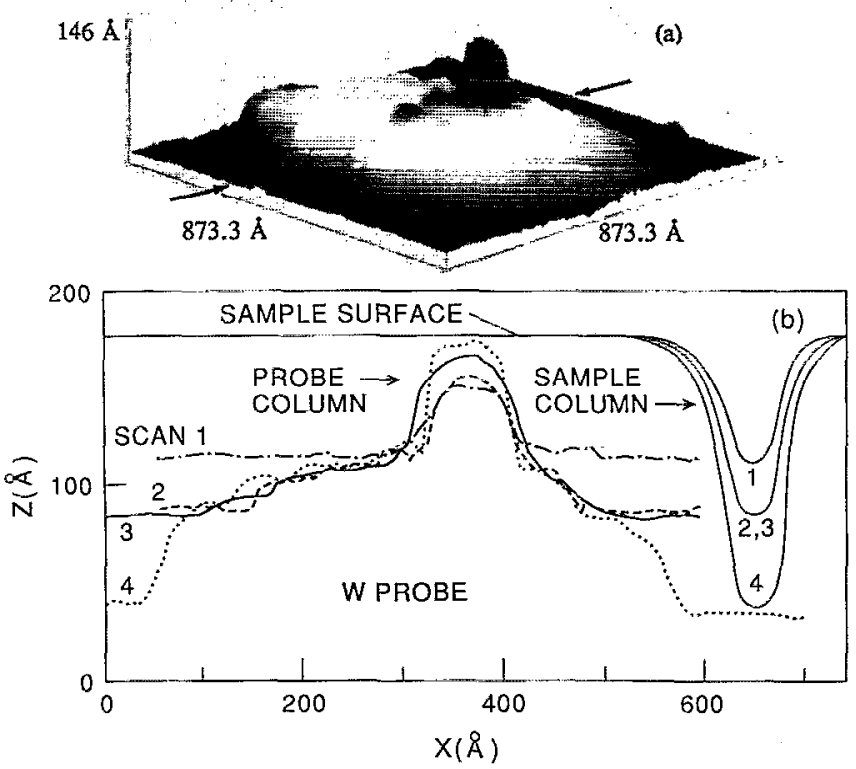

FIG. 1. (a) The image obtained after applying fourteen 3-7 V (sample positive), $1 \mathrm{~s}, 1 \mathrm{nA}$ feedback, voltage ramps at one position. (b) Four $Y$-direction scans between the arrows in part (a), taken between voltage ramps. Scan 1 was taken after one voltage ramp, scan 2 after 4 ramps, scan 3 after 11 ramps, and scan 4 after 14 ramps. The sample columns are shown to clarify interpretation of the images, and do not represent the actual column shapes.

sharp probe to initiate nanocolumn formation. The probe generally lengthens several hundred $\AA$ in this process and a $\sim 800 \AA$ region of the $\mathrm{Si}$ surface is severely damaged, indicating that $\mathrm{Si}$ has been added onto the $\mathrm{W}$ probe.

In Figs. 1 and 2 we show STM images of Si nanostruc-
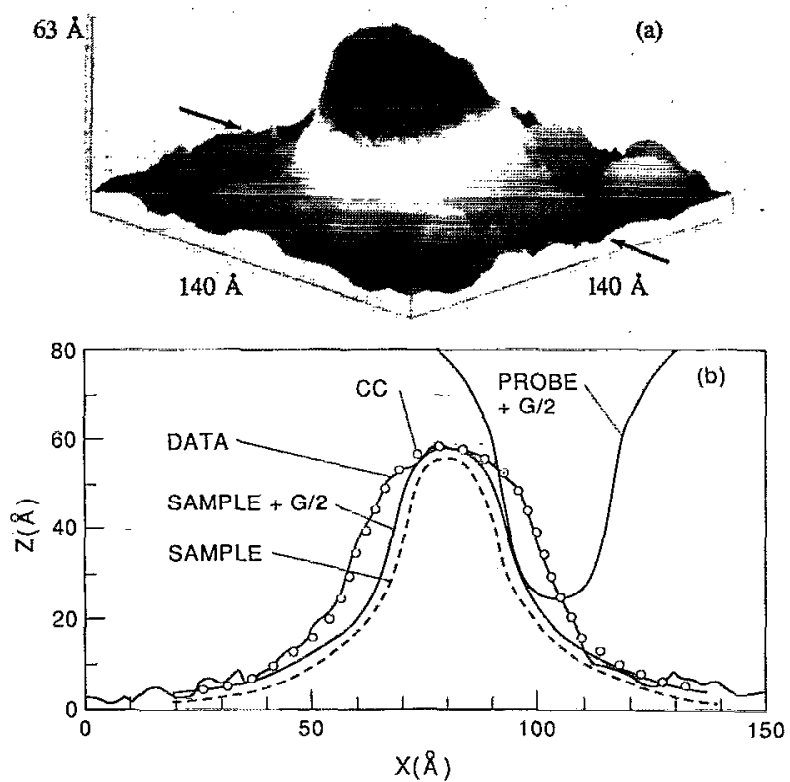

FIG. 2. (a) Image of a sample nanocolumn produced by applying $7 \mathrm{~V}$ at $1 \mathrm{nA}$ feedback for $10 \mathrm{~s}$. (b) An x-direction scan line (Data) through the nanocolumn in part (a) is compared to the contact convolution (open points) of the two solid line figures (Sample $+G / 2$ and Probe $+G / 2$ ). These represent proposed probe column and sample column plus $1 / 2$ of the tunneling gap $(G)$. The resulting sample surface obtained using $G=5$ $\AA$, is represented by the dashed line. tures produced by the construction steps given in the captions. Whereas STM scan pictures are almost always shown with highly expanded height scales, Fig. 2 shows the actual ratio of vertical to horizontal dimensions, and the vertical scale is expanded by a factor of 2 in Fig. 1. These STM images result from raster scans of the probe across the sample at constant tunneling current, so the recorded image is a contact convolution (CC) of the sample with the inverted surface of the probe at $\mathrm{a} \sim 5 \AA$ gap. For unequal-width structures protruding outward from the sample and probe surfaces, the image will primarily reproduce the broader of the two shapes. Standard probe etching preparations, as used here, yield irregular probes with 100$1000 \AA$ radius of tip curvature. Scanning such a probe across the sharp structures in these figures would simply reproduce the broad shape of the probe. Clearly, the above images can only be obtained once very narrow, columnlike structures (nanocolumns) are placed on both probe and sample.

When a relatively fiat topology is seen extending outward from the base of the nanostructure, this indicates that the probe nanocolumn is longer than that on the sample. As the sample nanocolumn increases in height with repeated pulses at one position, the image develops a broad base that is identified as the blunt end of the $W$ probe imaged by a longer sample nanocolumn. Such a sequence of images is shown in Fig. 1. Figure 1(a) shows a perspective view of the final image after repeated pulsing in one region, and Fig. 1(b) shows four scans across the structure, taken during its construction. The first scan, labeled 1 , shows a $33 \AA$ tall column, which is the height of the sample nanocolumn after one pulse. In the next scan the total feature has grown $68 \AA$ tall and acquired a broad base. This height above the plane is the new height of the sample column after the additional pulsing, and the broad base is the comparatively blunt end of the probe which is now visible. The narrow, $40 \AA$ tall structure on top is the probe nanocolumn. In scan 3 , the probe nanocolumn has grown $50 \AA$ tall and the sample column has not changed length. In scan 4, the probe nanocolumn has grown $57 \AA$ tall, while the sample nanocolumn has grown $140 \AA$ tall, exposing much more of the original probe. These probe column and sample column length changes are also detected as height changes above the plane and above the feature after each pulse, confirming these interpretations. The general character of these sample nanocolumns are drawn in the figure for insight. However, their widths in the base region are not known from these data, because the scan only shows the broader of the two opposing structures.

To indicate the typical shape of individual nanocolumns, we give an example of image analysis in Fig. 2(b), where a scan line through the image in Fig. 2(a) is shown. This image has a height of $58 \AA$ and a full width at half maximum (FWHM) of $45 \AA$. To indicate possible shapes of individual structures which might be responsible for this image, we have shown opposing columns with the same functional form and FWHM ( $31 \AA$ ) that yield a CC fit to the image. Since no pedestal is evident, the height of the 
sample column must equal the image height and the probe column must be taller. The shape of the sample column is relatively insensitive to the probe column length. In this example we use a probe column which is $30 \%$ larger than the sample column. For a $5 \AA$ tunneling gap the actual column "surfaces" are $2.5 \AA$ inside the surface of these contacted columns, as shown by the dashed line in the figure. The FWHM of this $58 \AA$ high sample column surface is thus $26 \AA$, where surface represents essentially the outer edge of the electron cloud of the surface atoms. In this example the probe column has the same width and a greater height than the sample column. If we had used a shorter probe column in the analysis both columns would be narrower. Any resistive voltage drop along the column will cause the tunneling gap to decrease toward the top, fattening the image. Increased contact area along the sides will similarly fatten the image compared to the actual column. A fitting using $58 \AA$ high columns and unequal widths will require that one column be narrower than 26 $\AA$. Thus, $26 \AA$ is an upper limit for the FWHM of the nanocolumn surface.

These nanostructures are believed to be made of $\mathrm{Si}$, based on the absence of anything but W and $\mathrm{Si}$ in sufficient quantity to be a candidate and on the changes in probe and sample column lengths that occur during construction (e.g., Fig. 1 discussion). Another indication is the fact that increased sample column height can be produced without major change in probe length or probe nanocolumn shape (e.g., Fig. 1). A predominance of $\mathrm{Si}$ atom motion is also consistent with the larger heat of vaporization of $\mathrm{W}$ and the large fields required for $\mathrm{W}$ field evaporation. ${ }^{13}$ Roughening and pitting of the nearby sample surface is observed (e.g., Fig. 2), increasing with repeated voltage pulsing in one region, as expected from sliding of $\mathrm{Si}$ atoms toward the probe nanocolumn.

The current-voltage relation on top of the nanocolumns is indistinguishable from that on the original sample surface and is characteristic of that observed using a freshly cleaned $\mathrm{W}$ probe and $\mathrm{Si}$ surface. This indicates that $<1 \mathrm{~V}$ drop occurs in the nanocolumn. This may be explained by ballistic electron transport, and also suggests well-ordered $\mathrm{Si}$ in the columns. In comparison, we have observed a much larger voltage drop across $150 \AA$ of intrinsic hydrogenated-amorphous-silicon film. ${ }^{14}$ The strength and current-carrying capacity (required to produce the STM image) of these very narrow, tall Si columns is very impressive and bodes well for Si-based nanoelectronics.

These nanocolumns are unchanged in height or shape by repeated scanning, or when rescanned after as long as $14 \mathrm{~h}$. However, three nanocolumns of $40-160 \AA$ height were scanned after $\sim 40 \mathrm{~h}$ of tunneling nearby and had lost 15-35 $\AA$ of height.

Several observations support a model of field-induced motion of Si atoms across the sample surface and up the growing column, sometimes accompanied by atoms hopping across the gap to the probe: The sample nanocolumn often grows $100-200 \AA$ tall without significant change in the probe length. Positive sample pulses are much more effective. Structures grow much faster and taller on the sample than on the probe. These results are consistent with Si surface atoms that have a positive dipole moment and are preferentially attracted to a negatively biased probe. However, we have also produced up to $35 \AA$ high sample columns with negative sample pulses, perhaps due to the polarizability of the surface $\mathrm{Si}$ atoms. Finally, the columns can only be made once the probe is sharp enough to induce radial fields on the sample surface. (With a blunt probe the fields are primarily vertical.)

The present observations provide a significant step toward STM-based nanotechnology. The Si nanocolumns produced here have great intrinsic interest for physics and electronics, and their amazingly thin yet robust shapes also bode well for the emerging field of nanotechnology. One can easily visualize constructing lithographic masks and many types of devices based on elaborations of these shapes. In addition, STM practitioners have long been plagued with difficult and unreliable probe preparations, and it has not been possible to produce a probe with the height/width required to observe tall nanostructures or deep etch pits. They will appreciate the outstanding value of a repeatable, in situ method of making tall, sharp probe extensions. We suspect that this new ability to measure the shapes of nanostructures that have previously been invisible will greatly accelerate progress in this field.

'G. M. Shedd and P. E. Russell, Nanotechnology 1, 67 (1990).

${ }^{2}$ J. A. Stroscio and D. M. Eigler, Science 254, 1319 (1991).

${ }^{3}$ D. M. Eigler and E. K. Schweizer, Nature 344, 524 (1990).

${ }^{4}$ J. A. Dagata, J. Schneir, H. H. Harary, C. J. Evans, M. T. Postek, and J. Bennett, Appl. Phys. Lett. 56, 2001 (1990).

${ }^{5}$ E. E. Ehrichs and A. L. de Lozanne, J. Vac. Sci. Technol. A 8, 571 (1990).

${ }^{6}$ E. E. Ehrichs, S. Yoon, and A. L. de Lozanne, Appl. Phys. Lett. 53, 2287 (1988).

${ }^{7}$ H. J. Mamin, P. H. Guethner, and D. Rugar, Phys. Rev. Lett. 65, 2418 (1990).

${ }^{8}$ R. S. Becker, J. A. Golovchenko, and B. S. Swartzentruber, Nature 325, 419 (1987).

${ }^{9}$ I.-W. Lyo and P. Avouris, Science 253, 173 (1991).

${ }^{10}$ D. M. Eigler, C. P. Lutz, and W. E. Rudge, Nature 352, 600 (1991).

${ }^{11}$ L. J. Whitman, J. A. Stroscio, R. A. Dragoset, and R. J. Celotta, Science 251, 1206 (1991).

${ }^{12}$ A. Ishizaka and V. Shiraki, J. Electrochem. Soc. 133, 666 (1986).

${ }^{13}$ T. T. Tsong, Surf. Sci. 70, 211 (1978).

${ }^{14} \mathrm{G}$. Stutzin, R. M. Ostrom, and A. Gallagher (unpublished). 